Gynäk. Rdsch. 1984;24:I-VI

\title{
Contents, Vol. 24, 1984
}

\section{Inhaltsverzeichnis Vol. 24,1984}

Nr. 1 Originalarbeiten

Anaerobier und Gardnerella bei «unspezifischem» Fluor vaginalis («Vaginose»)

Pavic, N.; Fankhauser-Mauri, C; Renggli, R.; Schwank, A 1

Bakterielle Besiedlung des Nabels und der Nase von Neugeborenen mit und ohne primären Vol. 24, 1984 Hautkontakt beim Anlegen unmittelbar post partum

Gerstner, G.; Gitsch, E.; Enzelsberger, H.; Metka, M.; Rotter, M 24

Geburtseinleitung durch intravaginale Applikation von Prostaglandin E2-haltigen Tabletten

Kofler, E.; Kubista, E.; Grünberger, W.; Husslein, P 31

Kurzfassungen von Zeitschriftenartikeln

Die Densitometrie als Hilfsmethode bei der Azetylcholinesterasebestimmung im Fruchtwasser

Goldfine, Ch.; Haddow, J.E.; Hudson, G.A.; Miller, W.A 43

Beziehung zwischen der Plazentareife im Ultraschallschnittbild und der L/S-Ratio im

Fruchtwasser

Tabsh, K.M.A 44

Postoperative Thromboseprophylaxe mit niedrig dosiertem Heparin bei gynäkologischen Malignomen: eine prospektive, kontrollierte Studie

Clarke-Pearson, D.L.; Coleman, R.E.; Synan, I.S.; Hinshaw, W.; Creasman, W.T. ... 45 Genitale

Hepes-simplex-Virus-Infektion bei schwangeren Frauen - Charakteristika und

Management der Schwangerschaft

Harger, J.H.; Pazin, G.J.; Armstrong, J.A.; Breinig, M.C.; Ho, M 47

Uteroplazentarer Durchblutungsindex bei intrauteriner Wachstumsretardation fetalen oder mütterlichen Ursprungs

Nylund, L.; Lunell, N.-O.; Lewander, R.; Sarby, B 49

Eisenbedarf bei normaler Schwangerschaft untersucht anhand von Serumferritin-, Serumtransferrinsättigungs- und Erythrozytenprotopo $\varphi$ hyrinbestimmungen

Romslo, I.; Haram, K.; Sagen, N.; Augensen, K 51

Phosphatidylglyzerol im Fruchtwasser: ein früher Indikator der fetalen Lungenreife

Whittle, M.J.; Wilson, A.I.; Whitfield, C.R 53

Die Verwendung von Gestagenen zur Nachbehandlung der operierten Endometriumkarzinome des Stadiums I

Moazzami, B.; Walt, J.D. van der; Boyd, N.R.H 54

Einfluss der äusseren Wendung des Fetus aus Beckenendlage in Schädellage in der Spätschwangerschaft auf die Sectiorate: eine kontrollierte Studie

Hofmeyr, G.J 56

Ergebnisse der Plazentadurchströmungsmessung bei Patientinnen mit schwangerschaftsbedingter Hypertonie

Voigt, R.; Stoll, W.; Estel, Ch 
Klinische Langzeitbeobachtung nach ovarieller Keilresektion bei polyzystisch ovariellem Syndrom

Hjortrup, A.; Kehlet, H.; Lockwood, K; Hasner, E 59

Abortusrate bei Schwangerschaften nach Ovaluationsauslösung durch Menopausengonadotropin/gonadotropes Chorionhormon

Ben-Rafael, Z.; Dor, J.; Mashiach, S.; Blankstein, J.; Lunenfeld, B.; Serr, D.M 60

Inhaltsverzeichnis

III

Fortbildung

Hormontherapie in der Nachsorge gynäkologischer Karzinome

Hormontherapie in der Nachsorge des Ovarialkarzinoms

Schönbauer, M.; Salzer, H 63

Hormontherapie in der Nachsorge des Endometriumkarzinoms

Salzer, H 66

Hormontherapie in der Nachsorge des Mammakarzinoms

Smekal, G.; Salzer, H 68

Strahlensensitivierende Wirkung von Medroxyprogesteron auf in vitro gezüchtete Endometriumkarzinomzellen

Huber, J $\quad 70$

Erratum $\quad 72$

Nr. 2 Originalarbeiten

Serumlipoproteine bei gesunden Schwangeren. Qualitative und quantitative Untersu-

chungen

Brockerhoff, P.; Holzer, A.; Schwenzer, E.; Rathgen, G.H 73

Die Vasektomie im Rahmen der Kontrazeption

Stamm, H.; Acheampong, A 85

Einfluss der Infektmorbidität auf die Fünfjahresheilung beim radikaloperierten Kollumkarzinom. Ergebnisse 1968-1978

Gerstner, GJ 98

Kurzfassungen von Zeitschriftenartikeln

Auswirkungen der Epiduralanalgesie auf die Funktion des unteren Hamtraktes im frühen

Wochenbett

Weil, A.; Reyes, H.; Rottenberg, R.D.; Béguin, F.; Herrmann, W.L 105

Supravaginale Uterusexstirpation im Vergleich zur Hysterektomie: Auswirkungen auf Koitusfrequenz und Dyspareunie

Kikku, P 107

Clomiphenzitrat als Therapie bei Korpus-Luteum-Insuffizienz

Downs, K.A.; Gibson, M 108

Wirkung von Danazol auf das pulsative Gonadotropinmuster und auf den Serumöstradiolgehalt bei normalzyklischen Patientinnen

Dmowski, W.P.; Headley, S.; Radwanska, E 110

Versagen von Bromocriptin bei der Wiederherstellung des Menstruationszyklus bei normoprolaktinämischer Amenorrhö nach Einnahme der Pille

Coehling Bennink, H.J.T.; van der Steeg, H.J 112

Volumetrische Selbstbestimmung des zervikovaginalen Sekrets: ein neuer Versuch der Ovulationszeitbestimmung 
Usala, St.J.; Schumacher, G.F.B 114

Tokolyse mit ß-Sympathikomimetika allein oder in Kombination mit dem Kalziumantagonisten Verapamil?

Carstensen, M.H.; Bahnsen, J.; Sterzing, E 115

Zytophotometrische Bestimmung der DNS bei minimal invasivem Zervixkarzinom beim

Menschen

Herzog, R.E.; Ciprian, C 117

Das Erleben der Geburt von Frauen mit und ohne Geburtsangst

Areskog, B.; Uddenberg, N.; Kjessler, B 119

Die Strahlentherapie des Endometriumkarzinoms

Frischkorn, R 120

Inhaltsverzeichnis

Fortbildung

Sitzung der Österreichischen Gesellschaft für Perinatologie, Innsbruck, 7. Mai 1983

Ursachen und Diagnostik der Frühgeburi

Lechner, W 122

Therapie und Geburtsleitung bei Frühgeburten

Bichler, A 124

Infektionsgefährdung Früh- und Neugeborener

Guggenbichler, J.P 127

Spätprognose unreifer Frühgeborener $(<1500$ g Geburtsgewicht $)$

Frisch, $\mathrm{H} \quad 129$

Varia 132

Originalarbeiten

Beurteilung der Plazentafunktion mit dem Plazentainsuffizienz-Score

Gitsch, E.; Philipp, K 133

Schwangerschaft nach wiederholten Aborten bei Uterusmissbildung

Schmid, J 140

Pyogene Sakroiliitis. Kasuistik und Review einer seltenen Komplikation im Wochenbett

Biedermann, K.; Schneider, K.T.M.; Kleinert, B.; Huch, A 145

Dermatologische und endokrinologische Aspekte des Hirsutismus

Schmidt, J.B.; Huber, J.; Spona, J 153

Therapeutische Möglichkeiten beim Hirsutismus

Huber, H.; Schmidt, J.B.; Spona, J 161

Kurzfassungen von Zeitschriftenartikeln

Phagozyten und Unfruchtbarkeit: Eileitermakrophagen als potentielle Mediatoren weib-

licher Sterilität

Haney, A.F.; Miskukonis, M.A.; Weinberg, J.B 168

Basaltemperaturkurvenauswertung: ein Vergleich zwischen verschiedenen Interpretations-

methoden

McCarthy, J.J.; Rockette, H.E170

Sexualhormonbindendes Globulin und Androgenkonzentrationen bei Diagnose und Therapie hirsuter Patienten

Cunningham, S.K.; Loughlin, T.; Culliton, M.; McKenna, T.J 172

Die Vorhersage fetaler Wachstumsretardation durch mütterliche HPL-Konzentrationen im Blut: Wahl eines Grenzwertes zwischen normal und abnormal 
Lilford, R.J.; Obiekwe, B.C.; Chard, T

Knochendichte bei amenorrhoischen Frauen mit und ohne Hyperprolaktinämie

Schlechter, J.A.; Sherman, B.; Martin, R 175

Das mikroinvasive Karzinom der Cervix uteri

van Nagell, J.R.; Greenwell, N.; Powell, D.F.; Donaldson, E.S.; Hanson, M.B.; Gay, E.C. 176

Verbesserte Therapiemöglichkeiten mit cisplatinhaltigen Zytostatikaschemata beim Ova-

rialkarzinom der Stadien HI und IV - durch Second-look-Operationen nachgewiesen

Cohen, CM.; Goldberg, J.D.; Holland, J.F.; Bruckner, H.W.; Deppe, G.; Gusberg, S.B.;

Wallach, R.C.; Kabakow, B.; Rodin, J 179

Zytoplasmatische Östrogen- und Progesteronrezeptoren beim primären Zervixkarzinom:

klinische und histopathologische Korrelate

Gao, Y.L.; Twiggs, L.B.; Leung, B.S.; Yu, W.C.Y.; Potish, R.A.; Okagaki, T.; Adcock, L.L.; Prem, K.A 180

Nachweis von Vitamin B12 bindenden Proteinen im Fruchtwasser zur Bestimmung der fetalen Reife

Grossowicz, N.; Jablonska, M.; Beyth, Y 182

Inhaltsverzeichnis

$\mathrm{V}$

Postpartales Vulvaödem nach der Entbindung im Gebärstuhl

Goodlin, R.C.; Frederick, LB 183

Laparoskopiebefunde bei Patientinnen mit Schmerzen im kleinen Becken

Cunanan, R.G.; Courey, N.G.; Lippes, J 185

Fortbildung

Erweiterte Abklärung endokrin bedingter Sterilitäten

Huber, H.; Spona, J 188

Die hyperprolaktinämische Ovarialinsuffizienz

Smekal, G.; Huber, H 191

Mikrochirurgische Operationen im Rahmen der Sterilitätsbehandlung

Philipp, K.; Huber, H 193

Buchbesprechung 196

Nr. 4 Originalarbeiten

Diabetes und Schwangerschaft

Schmid, J.; Kunz, J.; Sutter, R

Im Kardiotokogramm nachgewiesene, hyperreaktive Wehentätigkeit als frühes Zeichen einer symptomarm verlaufenden Abruptio placentae

Herrmann, U., jr.; Walther, M 210

Kurzfassungen von Zeitschriftenartikeln

Alphablocker zur Prophylaxe der Harnretention nach Hysterektomie

Livne, P.M.; Kaplan, B.; Ovadia, Y.; Servadio, C 215

Vaginalkarzinom nach Hysterektomie wegen Dysplasie oder Carcinoma in situ der Cervix uteri

Rasmussen, J.; Diernaes, E 216

Adenokarzinom des Endometriums im Stadium I: Behandlung, Überlebenszeit und Rezidive Philipsen, T.; Norgard, M 218

Zytosol und Kern-Östrogen- und -Gestagenrezeptoren sowie 17-Beta-Hydroxysteroiddehydrogenase im gesunden Gewebe und in benignen und malignen Tumoren des mensch- 
lichen Ovars

Vierikko, P.; Kauppila, A.; Vihko, R 220

Synchrone pulsierende Freisetzung von Prolaktin und LH bei hypogonadotropen Frauen

Cetel, N.D.; Yen, S.S.C 221

Fraktioniertes Kurzzeit-Afterloading mit hohen Dosisraten zur ambulanten Behandlung des inoperablen Korpuskarzinoms

Kucera, H.; Ünel, N.; Weghaupt, K 222

Der Effekt einer niedrig dosierten Heparinprophylaxe auf den Blutverlust bei abdominaler

Hysterektomie

Docherty, P.W.; Goodman, J.D.S.; Hill, J.G.; Pickles, B.G.; Boardman, J.; Taylor, C.G.;

Bush, P.V.; Skinner, L.E.M.; Slater, R.C 224

Zur Therapie der Stressinkontinenz mittels östrogenhaltiger Vaginalcreme

Hilton, P.; Stanton, S.L 226

Die Effektivität der hochdosierten Noräthisteron-Langzeitbehandlung bei Korpuskarzinompatienten. Ergebnisse einer prospektiven randomisierten Studie

Behling, H.; Krafft, W.; Schmeisser, G.; Schirmer, A.; Preibsch, W.; Brückmann, D. 228

Untersuchungen zur Rolle genitaler Mykoplasmen bei Frühgeburtlichkeit

Baumann, L.; Ruchkäberle, K.E.; Viehweg, B 229

Prolaktinsekretion bei Patienten mit Stein-Leventhal-Syndrom: Korrelation mit dem Ste-

roidmuster

Carmina, E.; Rosato, F.; Maggiore, M.; Gagliano, A.M.; Indovina, D.; Janni, A. ... 231

VI

Inhaltsverzeichnis

Fortbildung

Möglichkeiten und Grenzen gynäkologischen Operierens

10. Samstagsseminar der Frauenklinik im Klinikum Grosshadern der Universität

München, 5. November 1983 Ungewöhnliche Genitalprolapse

Albrich, W 234

Rezidivierende Harninkontinenz

Kümper, H.J.; Heinz, F.; Lentsch, P 237

Vaginale Fehlentwicklungen

Terruhn, V.; Götz, A 241

Tubare Sterilität

Götz, A.; Albrich, W.; Walz, C 244

Hysterektomie-Sektio

Eiermann, W 247

Typisches Vorgehen bei atypischen Operationen. Kurzreferat

Richter, K.; Wachter, I.; Tsoutsoulopulos, C 249

Autorenregister 253

Suppl. 1 Schweizerische Gesellschaft für Gynäkologie und Geburtshilfe

Bericht über die Jahresversammlung, 1.-4. April 1984 in St. Moritz

Société Suisse de Gynécologie et Obstétrique

Rapport de ГAssemblée ar > nuelle, 1 au 4 avril 1984 à St. Moritz

Suppl. 2 Jahrestagung der Osterreichischen Gesellschaft für Gynäkologie und Geburtshilfe

Juni 1984, Bad Mitterndorf

Suppl. 3 Immunotherapy of Vaginal Infections 
Scientific Papers of International Symposia in La Sarraz and Zurich, September 15/16, 1983 S. Karger · Verlag für Medizin und Naturwissenschaften — Basel · München — Paris · London · New York $\cdot$ Tokyo $\cdot$ Sydney

Dosierungsangaben von Medikamenten

Autoren und Verlag haben alle Anstrengungen unter-nommen, um sicherzustellen, dass Auswahl und Dosierungsangaben von Medikamenten im vorliegenden Text mit den aktuellen Vorschriften und der Praxis überein-stimmen. Trotzdem muss der Leser im Hinblick auf den Stand der Forschung, Änderungen staatlicher Gesetz-gebungen und den ununterbrochenen Fluss neuer Forschungsergebnisse bezüglich Medikamentenwirkung und Nebenwirkungen darauf aufmerksam gemacht werden, dass unbedingt bei jedem Medikament der Packungs-prospekt konsultiert werden muss, um mögliche Änderungen im Hinblick auf Indikation und Dosis nicht zu übersehen. Gleiches gilt fúr spezielle Warnungen und Vorsichtsmassnahmen. Ganz besonders gilt dieser Hin-weis fúr empfohlene neue und/oder nur selten gebrauchte Wirkstoffe.

Alle Rechte vorbehalten.

Ohne schriftliche Genehmigung des Verlags dürfen diese Publikation oder Teile daraus nicht in andere Sprachen übersetzt oder in írgendeiner Form mit mechanischen oder elektronischen Mitteln (einschliesslich Fotokopie, Tonaufnahme und Mikrokopie) reproduziert oder auf einem Datenträger oder einem Computersystem gespei-chert werden.

S. Karger AG, Postfach, CH-4009 Basel (Schweiz) Printed in Switzerland by Thür AG Offsetdruck, Pratteln 